Vacuum Gauge Control: The 338 VGC from Granville-Phillips offers a single display that provides direct pressure readout from above atmosphere to $1 \times 10^{-10}$ torr using a Convectron ${ }^{\mathrm{TM}}$ thermal conductivity gauge and a hot cathode ionization gauge. Infrequently used controls are housed behind a hinged front panel, and the 3-1/2-in.-high unit is available as a half-rack or as an 8-in.-wide benchmount unit. Crossover between the two gauges is automatic, and options include fourchannel process control for system automation, and both RS-232 and IEEE-488 computer interfaces for remote operation. Circle No. 77 on Reader Service Card.

Analytical Lab Training Plan: SAVANT Audiovisuals' Frequent Viewers Club allows members to view an unlimited number of analytical training programs for a one-time fixed fee. During the year-long membership, viewers may borrow an unlimited number of programs, one at a time and without additional cost. Programs include AA, ICP, GC, HPLC, IR, NMR, MS, UV-VIS, RIA, EIA, and lab safety. Most run about 20-45 minutes, and are available in slide/tape or video format for training groups or individuals. Members can also purchase programs at $50 \%$ off the current list price.

Circle No. 83 on Reader Service Card.

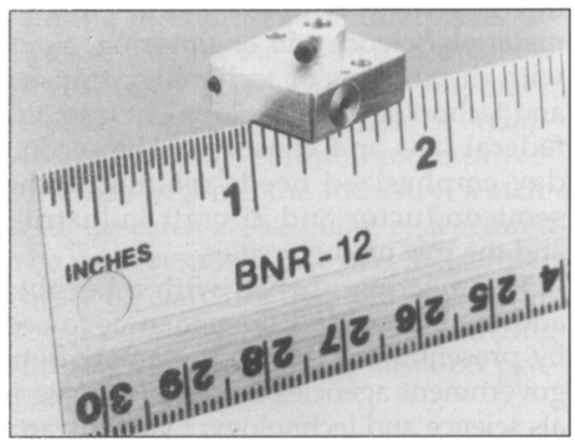

A

Miniature Rotation Stage: The 360 Micro-Rotation Stage from Charles Supper Company provides rotational adjustability for mounting components that fit into a $1 / 8$-in.-diameter mount hole. The micropositioner is constructed of aluminum with a hardened stainlesssteel socket drive, and full rotation is controlled by a six-spline drive key. The permanently lubricated device may be mounted, clamped, or epoxied into place, and comes with $0-80$ cap screws on $1 / 2-$ in. centers. Applications include mounting mirrors, beam splitters, and photo diodes, among others.

Circle No. 85 on Reader Service Card.

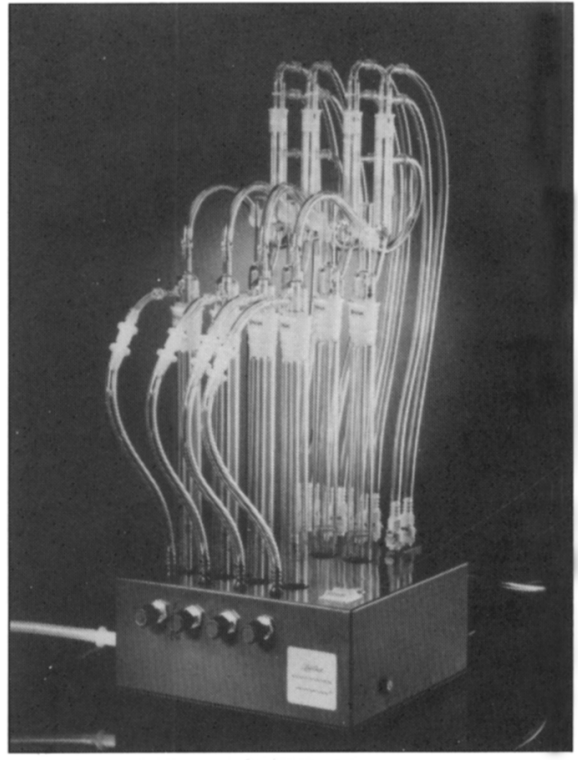

$\Delta$

Four-Station Distillation System: The Lab-Crest ${ }^{\oplus}$ Four-Station Midi Distillation System from Andrews Glass is composed of a distillation apparatus and a fourposition heat block and apparatus holder. The self-contained system can handle up to four $50-\mathrm{ml}$ liquid or sludge samples. The system's footprint is a 10-in. front panel x 10-3/4-in. side panel, with a net weight of $13 \mathrm{lbs}$. fully assembled. Applications include cyanide distillation and other analyses.

Circle No. 87 on Reader Service Card.

Modular Ion Pump Controller: Varian's MultiVac ${ }^{\mathrm{TM}}$ modular ion pump controller can simultaneously and independently run, monitor, and control two ion pumps. The user-programmable device is contained within a half-rack unit, and users may select variable or fixed voltage in any operating mode. The complete MultiVac $^{\mathrm{TM}}$ line offers a base unit, large and medium Vacion pump HV cards, a setpoint/remote control card, and computer interface. Applications include surface analysis and materials science, MBE, electron microscopy, high-energy physics, synchrotrons, and more.

Circle No. 89 on Reader Service Card.

Parallel Spectrometer: The Model 250PS from Chromex features multiple optical fiber inputs for characterizing emissions from diffuse or heterogeneous light sources such as plasmas, flames, or flows. A CCD video camera provides imaging and wavelength-resolved detection from the UV to NIR. A portable or desktop computer acquires data using propri- etary software with a frame grabber, and the system processes spectra for all channels and displays 3-D plots. Users control spectral range and resolution by means of a three-grating turret. Options include view control lenses for the fiber optic channels and cooled integration capability for the detector.

Circle No. 91 on Reader Service Card.

Microscope for Phase-Shift Mask Inspection: The MSM 100 Microlithography Simulation Microscope from Carl Zeiss allows users to characterize the operation of phase-shift masks prior to fabrication. Inspection and measurement can be carried out in the DUV to visible spectral range. Both aperture and image planes can be viewed for alignment, allowing the setting and control of the aperture ratio from 0.1 to 1 . Positioning of the phase-shift mask is performed by a motorized scanning stage that accommodates masks from 100-200 mm with front surface mounting, and with mask thickness ranging from $0.09-0.25$ in. Focusing is manual or motorized.

Circle No. 92 on Reader Service Card.

Liquid Helium Level Gauges: Each portable HLG200 Series gauge from Cryogenic includes a linear superconducting sensor to detect the interface between liquid and gaseous helium. An intermittent pulse with variable repetition rate minimizes helium consumption, and a high-current pulse drives the transition along the wire prior to each measurement. Each gauge also provides the facility for a linear percentage readout as an alternative to height readout in millimeters. Output can support a 4-20 mA current loop interface and measurements of up to 2 meters helium depth. Custommade probes are also available.

Circle No. 76 on Reader Service Card.

\section{Hot Isostatic Pressing Bibliography:}

The 325-page Bibliography on Hot Isostatic Pressing (HIP) Technology from the Metals Information Analysis Center of the Department of Defense lists 950 documents dealing with metals, alloys, and intermetallic compounds, and spans more than 450 materials. The bibliography is divided into three categories: powder metallurgy, casting, and miscellaneous materials. Within each category, information is organized according to major alloy groups, and each alloy group is further subdivided into individual commercial alloys.

Circle No. 88 on Reader Service Card. 